\title{
Differential Contribution of Frontal and Temporal Cortices to Auditory Change Detection: fMRI and ERP Results
}

\author{
Bertram Opitz,*,1 Teemu Rinne,† Axel Mecklinger,* D. Yves von Cramon,* and Erich Schrögerł \\ *Max Planck Institute of Cognitive Neuroscience, Leipzig, Germany; †Cognitive Brain Research Unit, Department of Psychology, \\ University of Helsinki, Hessinki, Finland; and ¥University of Leipzig, Leipzig, Germany
}

Received February 23, 2001

The present study addresses the functional role of the temporal and frontal lobes in auditory change detection. Prior event-related potential (ERP) research suggested that the mismatch negativity (MMN) reflects the involvement of a temporofrontal network subserving auditory change detection processes and the initiation of an involuntary attention switch. In the present study participants were presented with repetitive spectrally rich sounds. Infrequent changes of either small ( $10 \%$ change), medium ( $30 \%$ change), or large ( $100 \%$ change) magnitude were embedded in the stimulus train. ERPs and fMRI measures were obtained in the same subjects in subsequent sessions. Significant hemodynamic activation in the superior temporal gyri (STG) bilaterally and the opercular part of the right inferior frontal gyrus was observed for large and medium deviants only. ERPs showed that small deviants elicited MMN when presented in silence but not when presented with recorded MR background noise, indicating that small deviants were hardly detected under fMRI conditions. The MR signal change in temporal lobe regions was larger for large than for medium deviants. For the right fronto-opercular cortex the opposite pattern was observed. The strength of the temporal activation correlated with the amplitude of the change-related ERP at around $110 \mathrm{~ms}$ from stimulus onset while the frontal activation correlated with the change-related ERP at around $150 \mathrm{~ms}$. These results suggest that the right fronto-opercular cortex is part of the neural network generating the MMN. Three alternative explanations of these findings are discussed. $\odot 2002$ Elsevier Science

\section{INTRODUCTION}

Humans are able to rapidly reorient to changes in the auditory environment. On the basis of previous

\footnotetext{
${ }^{1}$ To whom all correspondence should be addressed at Max Planck Institute of Cognitive Neuroscience, P.O. Box 500355, D-04303 Leipzig, Germany. Fax: + 49341 9940113. E-mail: opitz@cns.mpg.de.
}

event-related potential (ERP) data, two separable systems have been proposed to explain auditory change detection (Näätänen, 1990). One, the "new afferentelements-activation" mechanism, detects transient changes in the physical properties of sounds via a differential state of refractoriness of the stimulus-specific neural populations. In the ERP this transientdetector mechanism is reflected in the N1 component. The second change detection mechanism registers differences between representations of an actual stimulus and of the invariancies inherent to the recent stimulation via a memory comparison process (Näätänen, 1990; Schröger, 1997) and is reflected in the mismatch negativity (MMN) (Näätänen et al., 1978). Both N 1 and MMN are generated mainly by the neural activity of sensory-specific systems in the vicinity of the auditory cortex.

Furthermore, it has been demonstrated that both mechanisms are involved in redirecting the focus of attention toward the sound changes. Escera et al. (1998) showed that auditory changes increase the distractibility in a visual forced-choice reaction time task. Interestingly, however, they found that MMN and N1 mechanisms had different behavioral effects: Small sound changes, which elicited only MMN, degraded performance accuracy by decreasing the hit rate, while novel sounds, generating large N1 waves, prolonged the reaction time in the same task. These differential distracting effects support the notion of two separate mechanisms of involuntary attention (Escera et al., 1998, 2000). Correspondingly, the relationship between MMN-generating processes and involuntary attention switching is supported by recent results of Schröger (1996). He demonstrated that tones deviating from a repetitive sequence of standard tones presented to the unattended ear elicited a MMN and prolonged reaction times in a subsequent intensity discrimination task for tones delivered to the other, attended ear. These findings support the view that the MMN-generating process can initiate involuntary switches of attention (Näätänen and Michie, 1979). 
Based on the first ERP experiments on deviancy processing revealing large scalp potentials at frontocentral recording sites, the hypothesis was formulated that the involuntary switch of attention might be subserved by the frontal lobes ( $N$ äätänen and Michie, 1979; see al so Näätänen, 1990). This view is supported by recent findings demonstrating that patients with dorsolateral prefrontal lesions show a marked impairment in attentional control of irrelevant sensory input (Chao and Knight, 1997), as well as a reduction of the $M M N$, especially over the lesioned hemisphere (Alain et al., 1989; Alho et al., 1994). Moreover, studies using current source density mapping reported an additional generator of the MMN presumably located in the right frontal lobes (Giard et al., 1990, but see Deouell et al., 1998, for bilateral frontal contribution). Recently, Rinne et al. (2000) estimated the source-current distribution as a function of time showing that the activity of the frontal MMN subcomponent was preceded by the activity of the temporal subcomponent. This frontal source was more prominent in the right than in the left hemisphere. Although these studies suggest that the right prefrontal lobe is involved in the MMN generation process, neither the precise frontal brain structures subserving change detection nor their functional significance in MMN processes are fully understood.

The present study addresses the issue taking advantage of the high spatial resolution provided by fMRI. According to previous ERP findings (cf. Näätänen and Michie, 1979) we hypothesize that the frontal lobe contribution to deviancy processing should be stronger the larger the informational significance, i.e., the degree of deviancy. Therefore, in the present study we compared brain responses elicited by three types of deviants systematically varying the degree of change.

\section{MATERIALS AND METHODS}

\section{Subjects}

The electrophysiological and hemodynamic brain responses were measured in 15 healthy right-handed volunteers (aged 22-27 years, 7 male). Due to excessive eyeblink artifacts in the EEG session two subjects had to be excluded from all ERP and fMRI analyses.

\section{Stimuli and Behavioral Procedure}

To facilitate MMN elicitation the stimuli were spectrally rich tones, consisting of three sinusoidal partials (cf. Tervaniemi et al., 2000b). The second and third components were 3 and $6 \mathrm{~dB}$, respectively, lower in intensity than the first component. Four different tones of 100-ms duration each were presented: a frequent $(P=0.88)$ standard tone consisted of frequencies
500, 1000, and $1500 \mathrm{~Hz}$. The infrequent deviant tones ( $P=0.04$ for each type) differed from the standard tone in frequency of all partials by 10,30 , and $100 \%$ change and are called small, medium, and large deviants, respectively, in the following. A total of 2440 stimuli were delivered binaurally via headphones at 70 and $85 \mathrm{~dB} /$ SPL for ERP and $\mathrm{fMRI}$ recordings, respectively. The onset-to-onset interval was 500 ms. The order of presentation was random, except that each deviant tone was preceded by at least six standard tones. Subjects were instructed to ignore the auditory stimuli and to attentively watch a silent cartoon video to be able to answer control questions after the experiment. The identical procedure was applied for both ERP and fMRI recordings.

\section{ERP Procedures}

EEGs and EOGs were recorded continuously from 64 electrode sites including the left mastoid using an Electrocap. The signals were online bandpass filtered $(0.1-70 \mathrm{~Hz})$ and digitized at $500 \mathrm{~Hz}$. Vertical and horizontal EOGs were recorded from two electrode pairs placed on the infra- and supraorbital ridges of the right eye and on the outer canthi of the two eyes. All leads were referenced to nose tip. Electrode impedance was kept below $2 \mathrm{k} \Omega$. ERP data were epoched off-line for a 500-ms period (including a 100-ms prestimulus baseline). Prior to averaging epochs were scanned for eye movement and other artifacts and were excluded from averaging when the standard deviation exceeded $40 \mu \mathrm{V}$. ERPs were computed separately for standard and deviant tones. The ERP component related to sound change was determined as the most prominent negative peak over frontocentral scalp areas in the 100-200 ms time window in the deviant minus standard difference waves of the grand average data across deviant types. Visual inspection of the data revealed that the change-related response el icited by the three deviant types was differentially affected by the transientdetector mechanism, reflected in the N1 component of the ERP. While the change-related response to small deviants comprised mainly the MMN component, that to large deviants consisted of enhanced N 1 (as compared with standards) and MMN. Therefore, the amplitude of the early (due to N1 and MMN contributions) and the late (only due to MMN) proportions of the change-related component were separately quantified as the mean voltage in an early (90-120 ms) and a late (140-170 ms) time window. Repeated-measures ANOVAs were used to compare the ERPs elicited by the three deviant types in both time windows. Greenhouse-Geisser correction was used where appropriate. Uncorrected degrees of freedom (df) and corrected $P$ values are reported in the Results. 


\section{MR Procedures}

I maging was performed with a $3 \mathrm{~T}$ Bruker Medspec $30 / 100$ system. A standard birdcage head coil was used. Subjects were supine on the scanner bed, with a stereotactic fixation system used to reduce head motion. In a separate session high-resolution wholebrain images were acquired to assist localization of activation foci using a 3D MDEFT (128 slice sagittal, 1.5-mm thickness, $256 \times 256$ voxel) (Lee et al ., 1995; Ugurbil et al., 1993). For each subject, conventional $\mathrm{T}_{1}$-weighted anatomic images (MDEFT: data matrix $256 \times 256$, TR 1.3 s, TE 10 ms (Norris, 2000; U gurbil et al., 1993)) in plane with the echo-planar images were acquired, to align the functional images to the 3D images. Finally, event-related functional images were recorded using a gradient-echo EPI sequence with a TE of $30 \mathrm{~ms}$, flip angle $90^{\circ}$, and TR of $1000 \mathrm{~ms}$ sensitive to blood oxygen level-dependent (BOLD) contrast. An acquisition volume consisted of eight axial slices parallel to the plane intersecting the anterior and posterior commissures (AC-PC plane). The matrix acquired was $64 \times 64$ with a field of view of $19.2 \mathrm{~mm}$, resulting in an in-plane resolution of $3 \times$ $3 \mathrm{~mm}$. The slice thickness was $5 \mathrm{~mm}$ with an interslice gap of $2 \mathrm{~mm}$. The most inferior slice was positioned $15 \mathrm{~mm}$ below the AC-PC plane. Five discarded volumes were acquired at the beginning of each run while tones were presented to allow stabilization of magnetization. Thus, a total of $1220 \mathrm{vol}-$ umes were acquired synchronously with the stimulation in two runs of 610 volumes each.

The data processing was performed using the software package LI PSI A (L ohmann et al., 2001; see al so http://www.cns.mpg.de/lipsia). Prior to statistical analyses motion artifacts were corrected using an affine rotation and translation correction (Kruggel et al., 1998). Second, low-frequency signal fluctuations were removed on a voxel-by-voxel basis (Kruggel et al., 1998). Finally, a spatial smoothing with a Gaussian kernel of two voxels was applied to emphasize spatially coherent activation pattern. The fMRI data were analyzed using a general linear model. The hemodynamic responses to the three different deviants and standards were modeled with a synthetic hemodynamic response function and its first-order temporal derivative (J osephs et al., 1997). The inclusion of the derivative accommodates for small shifts in the onset of the hemodynamic response (Friston et al., 1998). Responses to standard tones were randomly selected so that their number equals the number of responses to the deviants. Pairwise contrasts between the parameter estimates of the response gain for each deviant type and the standard were tested by voxelwise t tests separately for each subject. The t statistics were subsequently transformed to the unit normal $\mathrm{Z}$ distribution and transformed into the Talairach coordinate space (Talairach and Tournoux, 1988). Group statistics were calculated employing a random effects model. Based on a priori predictions (Celsis et al., 1999; Opitz et al., 1999a; Rinne et al., 2000) the specific differences in the superior temporal and right inferior frontal cortex reported below were considered significant when 10 or more contiguous voxels survived a threshold at $\mathrm{P}<0.01$ (cf. Henson et al., 1999).

In a second step, we examined the relationship between the BOLD response and the change-related ERP response. Therefore, the BOLD signal change across deviant types in significantly activated regions was extracted for each individual and subjected to a crosscorrelation analysis with the amplitude of the early and late proportions of the change-related ERP.

\section{RESULTS}

\section{ERP Measures}

All deviant types elicited a change-related negative deflection, peaking around 100-180 ms from stimulus onset, with a frontocentral distribution. The deviant-minus-standard difference waveforms (Fig. 1) show an increased amplitude and a decreased latency for large deviants as compared with medium and small deviants. Notably, this component to medium deviants had a double peak over frontal scalp locations, suggesting two subcomponents (see Fig. 1). F urthermore, the response to large deviants comprised mainly the earlier of the two peaks around $110 \mathrm{~ms}^{2}$ whereas the response to small deviants comprised mainly the later peak (around $160 \mathrm{~ms}$ ). A two-way repeated-measures ANOVA with factors deviant type (large, medium, small) and time window (90-120 ms, 140-170 ms) for the mean amplitude at $F Z$ and left mastoid (ML) electrodes revealed a main effect of deviant type (FZ: $F(2,24)=$ 4.09, $\mathrm{P}<0.05, \mathrm{ML}: \mathrm{F}(2,24)=7.27, \mathrm{P}<0.05)$ and a time window $\times$ deviant type interaction ( $F Z$ : $F(2,24)=10.68, P<0.001, M L: F(2,24)=12.69$, $P<0.001)$, confirming the view that two subprocesses contribute to the change-related ERP pattern. Tested separately for each time window a significant effect of deviant type was observed in the early but not in the late time window for both sites (FZ: $F(2,24)=17.06, P<0.001, M L: F(2,24)=13.83$, $\mathrm{P}<0.001)$. Furthermore, the change-related response to large deviants was followed by a P3a component indicating that a switch of attention had occurred (Näätänen, 1990; Schröger and Wolff, 1998).

\footnotetext{
${ }^{2}$ This earlier peak may comprise overlapping $\mathrm{N} 1$ and $\mathrm{MMN}$ contributions.
} 


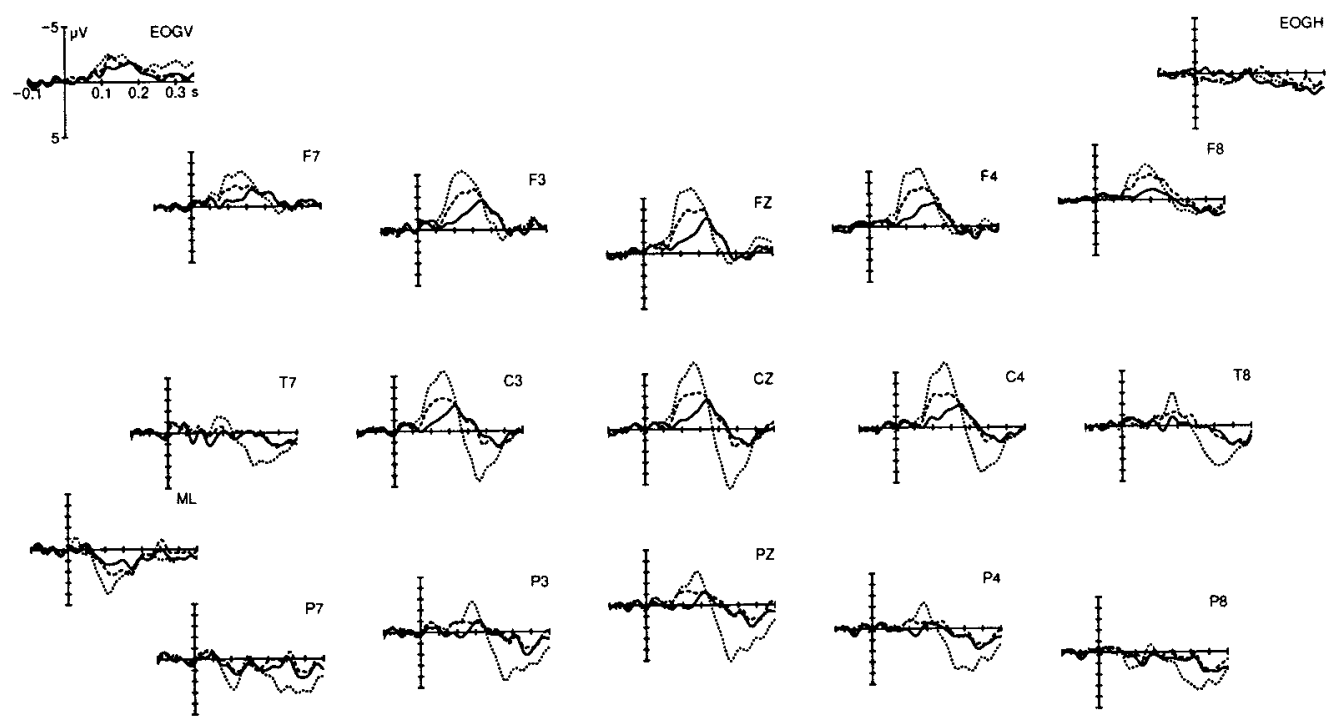

FIG. 1. Difference waveforms obtained by subtracting the ERPs to standard tones from those to the deviant tones separately are displayed for selected electrode sites. All sites were referenced to nose tip. Solid line, small deviants; dashed line, medium deviants; dotted line, large deviants.

\section{fMRI Measures}

The statistical parameter maps revealed significant bilateral activations in the superior temporal gyri ( $x, y$, z) of the Talairach (Talairach and Tournoux, 1988) space: $-61,-31,9$ and 58, -23, 9) for all deviant types. In addition, an activation of the opercular part of the right inferior frontal gyrus $(46,20,6)$ was observed. As apparent in Fig. 2 the temporal activity was largest for large deviants, whereas the frontal lobes exhibited greatest activation in response to medium deviants. These results point to a differential involvement of temporal and frontal regions in deviancy detection. This was confirmed by a repeated-measures ANOVA contrasting the mean signal changes of the three re gions of interest (ROIs) for the different deviant types, revealing a significant interaction of $\mathrm{ROI}$ and deviant type $(F(4,48)=4.69, P<0.01)$. Tested separately for the temporal lobe regions a trend toward a greater activity for large than for medium deviants in the left $(F(1,12)=3.93, P<0.1)$ and in the right $(F(1,12)=$ $4.28, \mathrm{P}<0.1)$ hemisphere was revealed. In the right fronto-opercular cortex medium deviants evoked stronger activity than large deviants $(F(1,12)=9.63, P<$ $0.01)$, whereas small deviants did not evoke a significant BOLD response in this brain structure.

\section{Follow-up ERP Experiment}

The finding that medium deviants evoked stronger activity than large deviants in the right frontal lobe was unexpected as it was hypothesized that the frontal activation increases with the stimulus deviance. The result that no frontal activity was detected in fMRI for the small deviants might be explained by the noise of the fMRI scanner which was not present in the ERP experiment. This hypothesis was tested in a follow-up ERP experiment in which the recorded fMRI noise was presented during the recording of the responses to the same stimuli. As in the main ERP experiment $13 \mathrm{vol}-$ unteers (8 from the main experiment and 5 new subjects) took part in this follow-up experiment. As apparent in Fig. 3 only large and medium deviants but not the small deviants elicited a robust MMN. ${ }^{3}$ This observation was confirmed first by an ANOVA contrasting the mean MMN amplitude at FZ of the three deviant types across ERP experiments (with noise vs without noise), revealing a main effect of deviant type $(F(2,26)=72.14, P<0.0001)$ and a significant deviant type $\times$ experiment interaction $(F(2,26)=6.83, P<$ $0.01)$. Tested separately for each deviant type, significant main effects of the experiment were obtained for small deviants only $(F(1,23)=11.34, P<0.005)$, indicating that the MMN to small deviants was substantially reduced when scanner noise is present. Moreover, no significant MMN to small deviants was elicited in the latter experiment as indicated by an ANOVA contrasting the mean amplitudes to standards and small deviants in both experiments, revealing a significant effect in the experiment without noise $(F(1,23)=$ $5.1, \mathrm{P}<0.01)$ but not in the experiment with noise ( $\mathrm{F}<$ 1). This indicates that the small, $10 \%$ deviation was not sufficiently processed to generate a reliable MMN or BOLD signal change when MR scanner noise was present. Therefore, the small deviant condition was

\footnotetext{
${ }^{3}$ Note that in this analysis no difference in the ERP pattern between the five new and eight old subjects was observed.
} 
large deviants

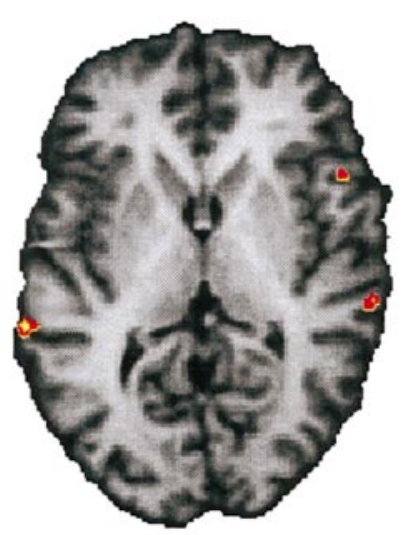

medium deviants

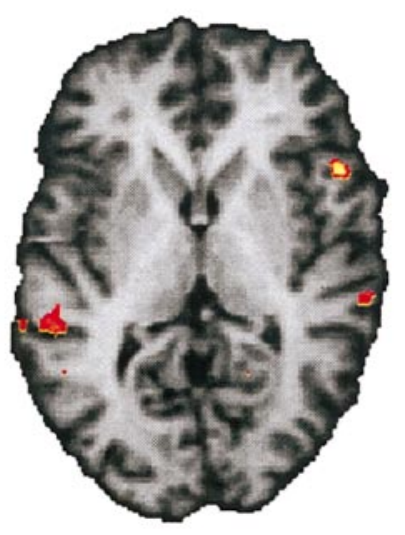

small deviants

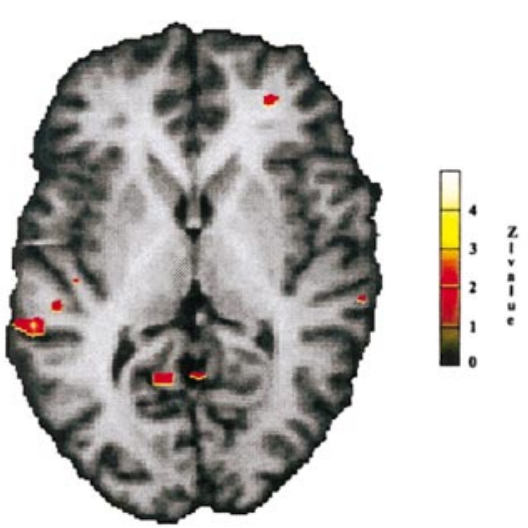

FIG. 2. Brain areas that showed significant $f M R I$ activation to the three deviant stimuli were superimposed on an individual structural magnetic resonance image in Talairach space. Images were thresholded at $P<0.01$. While all deviant types induced activation in the superior temporal gyri bilaterally (left: $-61,-31,9$; right: 58, $-23,9$ ), the opercular part of the right inferior frontal gyrus (46, 20, 6) was found active only for large and medium deviants.

excluded from all analyses comparing the electrical and hemodynamic responses to the deviants.

\section{Comparing ERP and $\mathrm{fMRI}$ Measures}

To compare the relationship between ERP and fMRI data, Pearson correlation coefficients between the amplitude of the two subcomponents of the change-related response at the $\mathrm{FZ}$ electrode in the initial ERP experiment and the BOLD signal change within each region were calculated. The correlation analysis for the large and medium deviants is displayed in Table 1.

The early part of the change-related ERP correlated with the signal change in the right superior temporal cortex. In addition, a significant correlation of the late portion of the change-related response with the signal change in the right inferior frontal gyrus was obtained. This might indicate a differential contribution of the two brain regions to the early and late proportions of
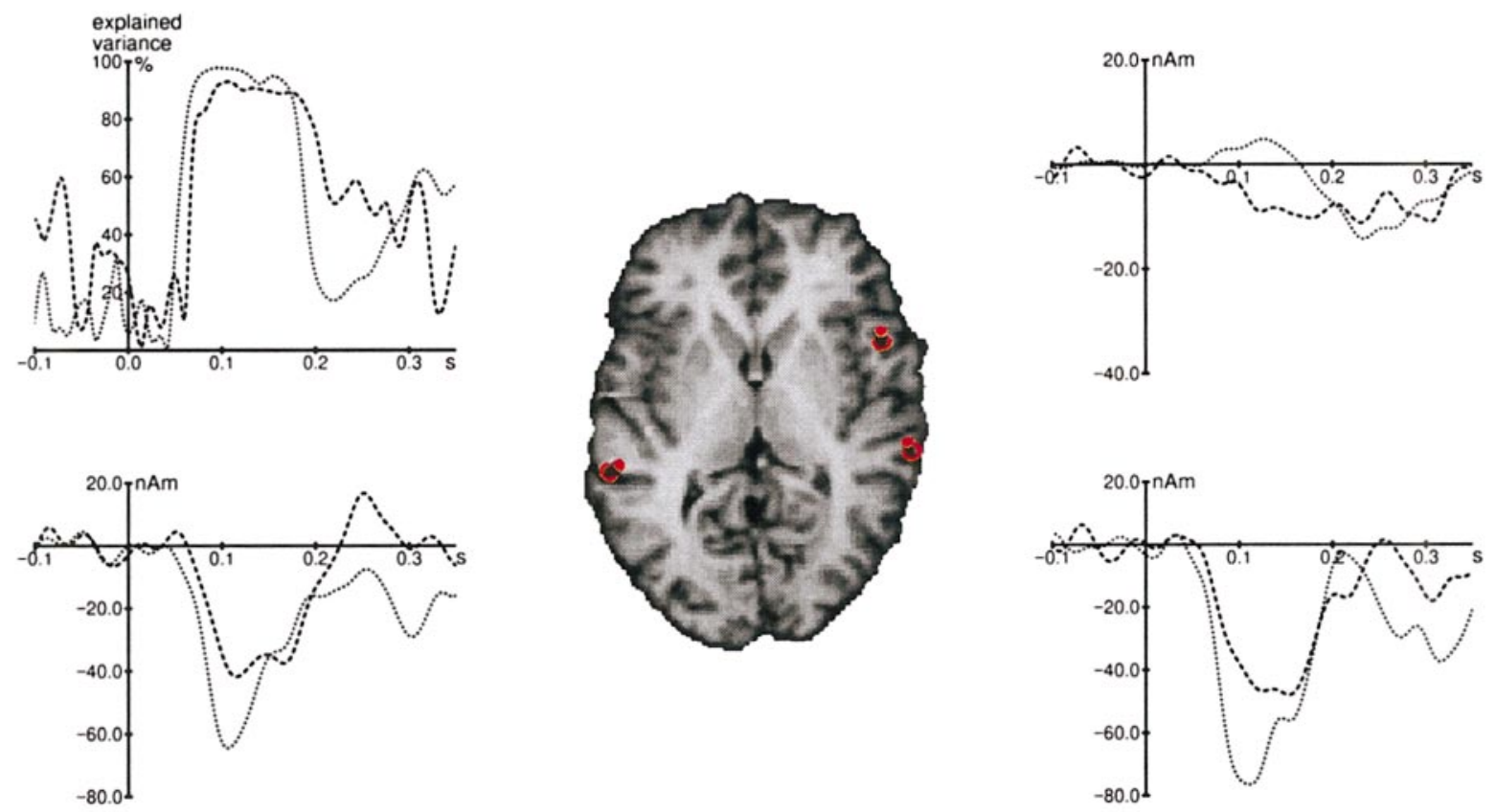

FIG. 4. Dipole locations (middle panel), explained variance (top left), and time course of dipole strength at left temporal (bottom left), right temporal (bottom right), and right frontal (top right) cortices for large and medium devants. For details see Fig. 1. 


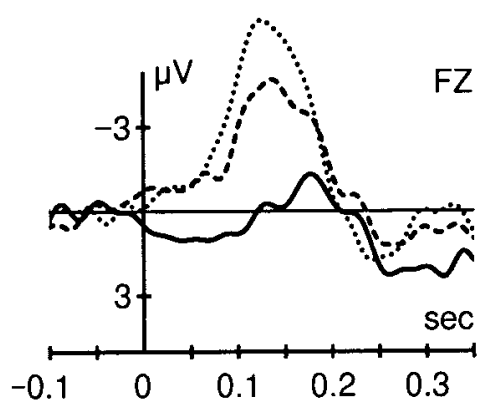

FIG. 3. MMN component elicited by large, medium, and small deviants when scanner noise is present. Difference waveforms were obtained by subtracting the ERPs to standard tones from those to all deviant tones separately. N ote that no statistically significant MMN was observed for small deviants. For details see Fig. 1.

the changerelated response, with the temporal cortex being more involved during the early period, i.e., the overlapping N 1/M MN component (cf. Alho et al., 1998; Opitz et al., 1999b), and the frontal lobe contributing to the generation of the MMN.

To further compare the ERP and $\mathrm{FMRI}$ results a dipole analysis was performed (cf. Opitz et al., 1999a,b). The locations of the dipoles were kept fixed according to the fMRI activation foci. Dipole orientations were fitted to explain the average reference ERP data using a realistically shaped head model (cf. ANT Software BV, Hengelo, The Netherlands). First, the scalp ERP distribution of large and medium deviants within the early time window (90-120 ms) was modeled with sources located in both temporal cortices. In a second step a third dipole placed in the right frontal operculum was added. This source configuration was used to model the ERPs in the late time window (140$170 \mathrm{~ms}$ ). As apparent in Fig. 4 (top left panel), the models for the large and medium deviants accounted for more than $95 \%$ of the signal variances in the time interval 94 to $172 \mathrm{~ms}$. The dipole activation strength was stronger for large deviants compared with medium deviants in the temporal cortex bilaterally. Interestingly, the reverse pattern, i.e., weaker dipole activity for large deviants than for medium deviants, was observed in right frontal cortex. Although the frontal source was not very reliable as it was difficult to separate it from the dominating temporal activation, these modeling results, together with the correlation analysis, point to a differential involvement of temporal and frontal brain areas in MMN generation.

\section{DISCUSSION}

The present study was aimed at identifying the precise brain structure in frontal lobes involved in auditory change detection and its role in these processes. Ther efore, three types of deviants systematically varying the degree of change were employed. All deviant types elicited a frontocentrally distributed change-re- lated response comprising mainly $\mathrm{MMN}$ (small deviants) and N 1/MMN (large deviants) portions. Furthermore, they significantly activated the bilateral superior temporal gyri. This is in agreement with previous PET and $F M R I$ findings as well as with recent MEG and ERP studies that localized the main generators of the MMN component in the vicinity of the auditory cortex (Alho et al., 1998; Opitz et al., 1999b; Scherg et al., 1989; Tervaniemi et al., 2000a). N otably, temporal activity, especially in the right hemisphere, increased with increasing degree of deviancy. Although this finding may, at least partially, be attributed to differential activation of the transient-detector mechanism by the three deviant types (cf. Tiitinen et al., 1994), it nevertheless points to a specific role of the right superior temporal cortex in the detection of nonphonetic auditory stimuli (Rinne et al., 1999; Tervaniemi et al., 2000a).

In addition to the activity in the temporal lobes, activation of the opercular part of the right inferior frontal gyrus was observed. To our knowledge, this is the first report of a frontal MMN generator directly observed using neuroimaging techniques. But, contrary to our hypothesis, the prefrontal activity was stronger for medium than for large deviants. This result can be accounted for in several ways:

The first explanation can be derived from the musictheoretical concept of pitch: tones belonging to the same pitch class are musically more similar than tones that are not (octave equivalence Burns, 1999). In the present experiment standard tones and large deviants were separated by an octave ( $100 \%$ change in all three partials) and, thus, despite their physical difference belong to the same pitch class. It could thus be assumed that the medium changes were perceived as more deviant than the large change and would, therefore, generate a stronger frontal MMN subcomponent. This interpretation is also in line with a recent MEG study demonstrating that the opercular part of the right inferior frontal gyrus specifically responds to violations of a musical expectancy (Maess et al., 2001).

\section{TABLE 1}

Coefficients of Pearson Correlations of the fMRI Activation in the Left and Right Temporal and Right Frontal Cortex with the Early and Late Subcomponents of the MMN at FZ

\begin{tabular}{lccc}
\hline & \multicolumn{3}{c}{ Signal change } \\
\cline { 2 - 4 } & Left & $\begin{array}{c}\text { Right } \\
\text { temporal }\end{array}$ & $\begin{array}{c}\text { Right } \\
\text { frontal }\end{array}$ \\
\hline Early response & $-0.12^{\mathrm{a}}$ & $-0.41^{*}$ & -0.20 \\
Late response & 0.04 & -0.15 & $-0.35^{*}$ \\
\hline
\end{tabular}

\footnotetext{
$* \mathrm{P}<0.05$.
}

a The negative correlation is due to the negative amplitude of the $M M N$; the larger the MMN component (i.e., the larger the negativity of the MMN in the ERP) the larger the increase in BOLD signal. 
Second, as demonstrated by previous studies (Näätänen, 1990; Schröger, 1996; Escera et al., 1998), large deviants are detected via the transient-detector mechanism (reflected in the N1) while small deviants do invoke the memory comparison process (reflected in the MMN). Therefore, it might be assumed that in the present study the medium deviant elicited a stronger fronto-opercular activation than the large deviants because the latter might be proccessed mainly (but not exclusively) by the N1 generating mechanism.

Third, previous findings have suggested a specific role of the right prefrontal cortex in tuning the sensory system according to processing demands (Roland, 1981, 1982). It might be possible that the frontal MMN generator is not directly connected to switching of attention, as previously suggested, but might be related to an involuntary amplification or contrast enhancement mechanism tuning the auditory change detection system. According to this third interpretation, activation of the frontal contrast enhancement system increases with decreasing distinctiveness of sound input, as in a noisy environment like a MR scanner.

Taken together the present data provide the first direct evidence for a frontal MMN generator located in the opercular part of the right inferior frontal gyrus. Moreover, they clearly demonstrate that this frontal component can be influenced by the degree of deviation, thereby providing a starting point to further elucidate the functional role of the right fronto-opercular cortex in deviancy processing.

\section{ACKNOWLEDGMENTS}

This work was supported by the Leibniz Prize of the German Research Foundaton awarded to Angela D. Friederici, Director at the Max Planck Institute of Cognitive Neuroscience. We thank Björn Helmer Schmidt for helpful comments on earlier versions of the manuscript.

\section{REFERENCES}

Alain, C., Richer, F., Achim, A., and Saint-Hilaire, J . M. 1989. Human intracerebral potentials associated with target, novel and omitted auditory stimuli. Brain Topogr. 1: 237-245.

Alho, K., Winkler, I., Escera, C., Huotilainen, M., Virtanen, J., J ääskeläinen, I. P., Pekkonen, E., and IImoniemi, R. J. 1998. Processing of novel sounds and frequency changes in the human auditory cortex: Magnetoencephalographic recordings. Psychophysiology 35: 211-224.

Alho, K., Woods, D. L., Algazi, A., Knight, R. T., and Näätänen, R. 1994. Lesions of frontal cortex diminish the auditory mismatch negativity. Electroencephalogr. Clin. Neurophysiol. 91: 353-362.

Burns, E. M. 1999. Intervals, scales, and tuning. In The Psychology of Music (D. Deutsch, Ed.), pp. 215-264. Academic Press, San Diego, CA.

Celsis, P., Boulanouar, K., Doyon, B., Ranjeva, J. P., Berry, I., Nespoulous, J . L., and Chollet, F. 1999. Differential fMRI responses in the left posterior superior temporal gyrus and left supramarginal gyrus to habituation and change detection in syllables and tones. Neurol mage 9: 135-144.
Chao, L. L., and Knight, R. T. 1997. Human prefrontal lesions increase distractibility to irrelevant sensory inputs. NeuroReport 6: 1605-1610.

Deouell, L., Bentin, S., and Giard, M. H. 1998. Mismatch negativity in dichotic listening: Evidence for interhemispheric differences and multiple generators. Psychophysiology 35: 355-365.

Escera, C., Alho, K., Schröger, E., and Winkler, I. 2000. Involuntary attention and distractibility as evaluated with event-related brain potentials. Audiol. Neurootol. 5: 151-166.

Escera, C., Alho, K., Winkler, I., and Näätänen, R. 1998. Neural mechanisms of involuntary attention to acoustic novelty and change. J . Cogn. Neurosci. 10: 590-604.

Friston, K., Fletcher, P., J osephs, O., Holmes, A., Rugg, M. D., and Turner, R. 1998. Event-related fMRI: Characterizing differential responses. Neurol mage 7: 30-40.

Giard, M. H., Perrin, F., Pernier, J ., and Bouchet, P. 1990. Brain generators implicated in processing of auditory stimulus deviance: A topographic event-related potential study. Psychophysiology 27: 627-640.

Henson, R. N., Rugg, M. D., Shallice, T., J osephs, O., and Dolan, R. J . 1999. Recollection and familiarity in recognition memory: An event-related functional magnetic resonance imaging study. J . Neurosci. 19: 3962-3972.

J osephs, O., Turner, R., and Friston, K. 1997. Event-related fMRI. Hum. Brain Mapping 5: 243-248.

Kruggel, F., Descombes, X., and von Cramon, D. Y. 1998. Preprocessing of fMRI datasets. In Workshop on Biomedical I mage Analysis (B. Vemuri, Ed.), pp. 323-330. IEEE Pre Los Angeles.

Lee, J .-H., Garwood, M., Menon, R., Adriany, G., Andersen, P., and Truwit, C. L. 1995. High contrast and fast three-dimensional magnetic resonance imaging at high fields. Magn. Reson. Med. 34: 308.

Lohmann, G., Mueller, K., Bosch, V., Mentzel, H., Hessler, S., Chen, L., and von Cramon, D. Y. 2001. A new software system for the evaluation of functional magnetic resonance images of the human brain. Comput. Med. Imaging Graphics, in press.

Maess, B., Koelsch, S., Gunter, T., and Friederici, A. D. 2001. Musical syntax is processed in the area of broca: An MEG-study. Nat. Neurosci. 4: 540-545.

Näätänen, R. 1990. The role of attention in auditory information processing as revealed by event-related potentials and other brain measures of cognitive function. Behav. Brain Res. 13: 201-288.

Näätänen, R., Gaillard, A. W. K., and Mäntysalo, S. 1978. Early selective attention reinterpreted. Acta Psychol. 42: 313-329.

Näätänen, R., and Michie, P. T. 1979. Early selective attention effects on the evoked potential: A critical review and reinterpretation. Biol. Psychol. 8: 81-136.

Norris, D. G. 2000. Reduced power multi-slice MDEFT imaging. J . Magn. Reson. Imaging 11: 445- 451.

Opitz, B., Mecklinger, A., von Cramon, D. Y., and Kruggel, F. 1999a. Combining electrophysiological and hemodynamic measures of the auditory oddball. Psychophysiol. Speci. Rep. 36: 142-147.

Opitz, B., Mecklinger, A., Friederici, A. D., and von Cramon, D. Y. 1999b. The functional neuroanatomy of novelty processing: Integrating ERP and fMRI results. Cereb. Cortex 9: 379-391.

Rinne, T., Alho, K., Alku, P., Holi, M., Sinkkonen, J ., Virtanen, J ., Bertrand, O., and Näätänen, R. 1999. Analysis of speech sounds is left-hemisphere predominant at 100-150 ms after sound onset. NeuroReport 10: 1113-1117.

Rinne, T., Alho, K., Ilmoniemi, R. J ., Virtanen, J ., and Näätänen, R. 2000. Separate time behaviors of the temporal and frontal mismatch negativity source. Neurol mage 12: 14-19.

Roland, P. E. 1981. Somatotopical tuning of postcentral gyrus during focal attention in man: A regional cerebral blood flow study. J. Neurophysiol. 46: 744-754. 
Roland, P. E. 1982. Cortical regulation of selective attention in man: A regional cerebral blood flow study. J . Neurophysiol. 48: 1059-1077.

Scherg, M., Vajsar, J ., and Picton, T. W. 1989. A source analysis of the late human auditory evoked potentials. J . Cogn. Neurosci. 1: 336-355.

Schröger, E. 1996. A neural mechanism for involuntary attention shifts to changes in auditory stimulation. J . Cogn. Neurosci. 8: 527-539.

Schröger, E. 1997. On the detection of auditory deviations: A preattentive activation model. Psychophysiology 34: 245-257.

Schröger, E., and Wolff, C. 1998. Behavioral and electrophysiological effects of task-irrelevant sound change: A new distraction paradigm. Cogn. Brain Res. 7: 71- 87.

Talairach, J ., and Tournoux, P. 1988. Co-planar Stereotaxis Atlas of the Human Brain. Thieme, New York.
Tervaniemi, M., Medvedev, S. V., Alho, K., Pakhomov, S. V., Roudas, M. S., van Zuijen, T. L., and Näätänen, R. 2000a. Lateralized automatic auditory processing of phonetic versus musical information: A PET study. Hum. Brain Mapping 10: 74-79.

Tervaniemi, M., Schröger, E., Saher, M., and Näätänen, R. 2000b. Effects of spectral complexity and sound duration on automatic complex-sound pitch processing in humans: A mismatch negativity study. Neurosci. Lett. 290: 66-70.

Tiitinen, H., May, P., Reinikainen, K., and Näätänen, R. 1994. Attentive novelty detection in humans is governed by pre-attentive sensory memory. Nature 372: 90-92.

Ugurbil, K., Garwood, M., Ellermann, J ., Hendrich, K., Hinke, R., Hu, X., Kim, S.-G., Menon, R., Merkle, H., and Ogawa, S. 1993. I maging at high magnetic fields: I nitial experiences at 4T. Magn. Resona. Q. 9: 259. 